\title{
A Comprehensive Review on Adiabatic Switching Circuits
}

\author{
Deepti Shinghal ${ }^{1}$, Farooq Husain ${ }^{2}$, Shuchita Saxena ${ }^{1}$, Amit Saxena ${ }^{1}$, Kshitij Shinghal ${ }^{3}$ \\ ${ }^{1}$ Asstt. Prof., ${ }^{2}$ Assoc. Prof. \& Head, ${ }^{3}$ Assoc. Prof. \\ Deptt. of E\&C Engg., MIT, Moradabad
}

\begin{abstract}
The technological evolution has increased the number of transistors for a given die area significantly and increased the switching speed from few $\mathrm{MHz}$ to GHz range. This leads to requirements of low power circuits. With the recent developments there have been a lot of technological evolutions one such evolution is adiabatic switching circuits. It should be noted that for adiabatic operation of the circuit is an ideal condition which may only be approached asymptotically as the switching process is slowed down. The signal energies stored in the circuit capacitances are recycled instead, of being dissipated as heat.
\end{abstract}

KEYWORDS: Adiabatic, Reversible Logic, ASIP, ASIC, Instruction set

\section{INTRODUCTION}

Designing electronic systems using digital techniques has become a well-accepted standard with electronic design community. The precision and speed of any computational block in digital design can be increased by just widening the data bus and making computational blocks compatible with widened data bus [1]. Thus a complex digital electronic system handles wide data buses, addresses large memory space and embeds the logical and computational blocks with appropriate data bits to match the widened data bus $[2,3,4]$. Due to the typical features of CMOS technology, for integrating complex systems in small silicon area with fairly low power dissipation at speeds demanded by most of the applications, CMOS VLSI has been the obvious choice of designers [5,6]. About $90 \%$ of the total electronic systems use CMOS technology at the back end. Then, to limit the power dissipation, alternative solutions at each level of abstraction are proposed. In present work attempts are made to review adiabatic logic circuits. The present work focuses on carefully studying adiabatic developments [7,8]. The study and survey of the different types of adiabatic logic circuits is carried out. The study survey and comparison of the different types of CMOS based logic circuits with adiabatic logic circuits is also done. In literature, there are two kinds of adiabatic circuits presented one is full-adiabatic and other is quasi-adiabatic or partial adiabatic circuits $[9,10]$. Rest of the paper is organized as follows the section to covers related work whereas section finally section 3 covers conclusion and future research directions.

\section{RELATED WORK}

Paul Leroux, et.al. gave Design and Assessment of a Circuit and Layout Level Radiation Hardened CMOS VCSEL Driver. They gave the radiation laborious style of a $155 \mathrm{Mb} / \mathrm{s}, 0.7 \mathrm{~m}$ CMOS driver for a verticalcavity surface-emitting laser (VCSEL). The circuit options increased tolerance to radiation evoked shifts within the device characteristics by using a replica-based feedback mechanism. The layout was achieved 


\section{International Journal of Recent Trends in Electrical \& Electronics Engg., Apr., 2018. CIJRTE \\ ISSN: 22316612}

with the help of an in-house developed radiation hardened part library. At an occasional rate of $4.5 \mathrm{~Gy} / \mathrm{h}$ or $450 \mathrm{rad} / \mathrm{h}$, the output current remains constant up to a minimum of $3.5 \mathrm{kGy}$. At a rate of $21 \mathrm{kGy} / \mathrm{h}$, the output current of the motive force drops by $100 \%$ at a dose of $3.5 \mathrm{MGy}$ and breaks down utterly at 5.5 MGy [12].

Diego Mateo and Antonio Rubio in 1998 proposed a design and Implementation of a Multiplier in a QuasiAdiabatic Ternary CMOS Logic. Adiabatic switch could be a technique to style low power digital IC's. Totally adiabatic logics have costly silicon space necessities. To resolve this disadvantage, a quasi-adiabatic ternary logic is projected. Its basis is conferred, Results show a satisfactory power saving with regard to standard and alternative quasi-adiabatic binary multipliers, and a decrease of the area required with regard to a completely adiabatic binary one [13].

Joohee Kim Conrad et.al. In 2002 suggested an Energy Recovering Static Memory suggested an energyrecovering adiabatic static RAM with a unique driver that moderates power dissipation by effectively recovering energy from the bit/word line capacitors. Energized by a single-phase ac power-clock, the proposed SRAM delivers browse and write operations with single-cycle latency. Thereto finish, a precharge-low scheme is employed along with a modified sense amplifier design that achieves high efficiency at differential voltages. Feedback circuitry from the driver output to the control circuit ensures that our driver remains efficient, not dependent on of the access pattern. The energy convalescent SRAM functions properly whereas achieving substantial energy savings over a large spread of clock voltages and operative frequencies. $\mathrm{H}$ zest recreations of a direct full custom adiabatic 256x256 SRAM, that has the vitality recouping bit/word line drivers, the cell cluster, and the sense amplifiers, appear more than $2.6 \mathrm{x}$ vitality reserve funds at $3 \mathrm{~V}, 300 \mathrm{MHz}$ as contrasted and its standard partner [14]. Energy Transfer and Recovery Efficiencies for Adiabatic Charging with Various Driving Waveforms was given by Michael P. Frank and Marco Ottavi in 2006. A standard drawback that arises within the study of energy-recovering circuits for digital logic is to investigate the relative energy potency of various attainable voltage waveforms for the power/clock signals that are accustomed drive adiabatic charge transfers within the logic. To well characterize these wave form potency problems provides an analytical tool that's useful within the style improvement of economical power/clock signal generators. during this note, acceptable metrics for the comparison of the energy efficiencies of various voltage waveforms within the context of necessities on the energy transferred to the logic load or the energy provided by the power/clock resonator, and show however these potency metrics scale as functions of frequency for a variety of easy wave shapes during a basic lumped-element circuit model [15].

Prasad D Khandekar, et al. in year 2010 suggested Implementation and Analysis of Quasi-Adiabatic Inverters. They mentioned the implementation of 3 quasi-adiabatic logic designs and analyzes the charge flow. All the NOT gate circuits are styled with the help of $180 \mathrm{~nm}$ technology in Cadence design setting [16].

Ke Wu, et.al. Designed a Low-Voltage True-Single-Phase-Clocking (TSPC) Logic using Bulk Dynamic Threshold MOS Technique. Their work concerned a replacement quick Bulk True Single phase duration (TSPC) based scheme. Dynamic Threshold MOS technique for each NMOS and PMOS was introduced. In planned methodology the common substrate of the NMOS logic or PMOS logic was dynamically controlled: the potential changes if only these transistors ought to work and keeps high threshold after they are stop working. And also the theme uses the charge recovery technique of the substrate to more scale back power. It's capable of in operation at $0.8 \mathrm{~V}$ or maybe lower. The planned theme is shown to be $33.45 \%$ quicker and has $20.86 \%$ energy savings compared to the regular TSPC logic circuits, throughout HSPICE simulation [17].

Prasad D Khandekar, and Dr. Mrs. Shaila Subbaraman gave a novel architecture for Low Power 2:1 MUX for Barrel Shifter. Barrel Shifter is a very important block within the processor design and not a lot of effort has been done to reduce its power dissipation. A barrel shifter wants n MUX for n-bit shifting and thus planning a MUX for low power to use it as a repetitive block within the barrel shifter can significantly cut back the simulation time. The end result of the analysis provided pointers for planning barrel shifter with the help of immoderate low power MUX [18]. 


\section{International Journal of Recent Trends in Electrical \& Electronics Engg., Apr., 2018. OIJRTE \\ ISSN: 22316612}

Prasad D Khandekar and Shaila Subbaraman suggested in another paper a Low Power Inverter and Barrel Shifter Design using adiabatic principle, since most of the scientific applications need floating point computations that need barrel shifter as the major element for hardware implementation and since not a lot of efforts are according to be taken by the researchers to attenuate its power dissipation, it's planned to implement barrel shifter with the help of adiabatic logic. A barrel shifter wants $n \log n$ multiplexers for $n-$ bit shifting. Therefore planning a low power MUX for employing as a repetitive block within the barrel shifter can significantly cut back the power dissipation in barrel shifter. The simulation results compared the power and delay parameters of basic multiplexer with the help of typical CMOS approach and adiabatic logic approach [19].

Tadahiro Kuroda and Mototsugu Hamada proposed Low-Power CMOS Digital Design with Dual Embedded Adaptive Power Supplies. In their work they given a low-power CMOS style methodology with twin embedded adaptive power supplies. It's found that the lower supply voltage ought to be set at 0.7 of the upper supply voltage to attenuate chip power dissipation. This information aids researchers in deciding the optimum supply voltages inside a restricted design time. An MEPG-4 video codec chip is intended at 2.5 and $1.75 \mathrm{~V}$ for internal circuits that are generated from an external power supply of $3.3 \mathrm{~V}$ by the dualVS circuits. Power dissipation is reduced by 57 while not degrading circuit performance compared to a traditional CMOS style [20].

Low-Power, Low-Noise Adder Design with Pass-transistor Adiabatic Logic was proposed by Hamid Mahmoodi-Meimand and Ali Afzali-Kusha. The potency of a completely adiabatic logic circuit is compared with its combinative and pipelined static CMOS counterparts. An 8-bit carry look-ahead adder id developed employing a 0.6-pm CMOS technology for all 3 logic designs. Supported on the post-layout simulation results, the adiabatic adder exhibits energy savings of $76 \%$ to eighty seven $\%$ and eighty seven $\%$ to $90 \%$ compared to its combinative and pipelined static CMOS counterparts, severally. It conjointly exhibits a substantial reduction in switching noise, compared to its static CMOS counterparts [21].

Joonho Lim, etal proposed NMOS Reversible Energy Recovery Logic for Ultra-Low-Energy Applications. It is a completely reversible adiabatic logic, NMOS reversible energy recovery logic (NRERL), that uses NMOS transistors solely and an easier 6-phase clocked power. Authors expressed that at low-speed operation, space overhead and energy consumption are smaller, compared with the other totally adiabatic logics. Bootstrapped NMOS switches are utilized to alter the NRERL circuits [22].

Balaji Narasimham, etal developed a new on-chip single-event transient (SET) check structure to autonomously characterize the widths of random SET pulses. Simulation results showed that the measure coarseness rapidly scales down with technology. Conjointly the experimental results indicated pulse widths variable from regarding $900 \mathrm{psec}$ to over3 nsec because the laser energy was enhanced [23].

Samik Samanta gave in his work a Power Efficient VLSI Inverter Design using Adiabatic Logic and Estimation of Power dissipation using VLSI-EDA Tool, he applied adiabatic logic style approach to style COMS NOT Gate. Author designed and simulated PFAL NOT gates and power dissipation is compared with the static CMOS inverter [24].

Massimo Alioto and Gaetano Palumbo recommended Power Estimation in adiabatic Circuits: a simple and accurate Model, they planned a technique supported by a linearization of the circuit and simplifying the analytical result obtained on the equivalent network. The approach ends up in easy relationships which may be used for a pencil-and-paper analysis or enforced on computer code. The accuracy of the results is validated by means that of Spice simulations on an adiabatic full adder designed with a $0.8 \mathrm{~m}$ technology [25].

Shun Li et.al presented a new technique Quasi-Static Energy Recovery Logic with Single Power-Clock Supply. Authors presented a new Quasi-Static Single part Energy Recovery Logic (QSSERL) that, not like the other existing adiabatic logic family, uses single ac supply-clock without extra voltages. This not solely ensures lower energy dissipation, however conjointly simplifies the clock style which might be otherwise additional difficult as a result of the signal synchronization demand. It's demonstrated that QSSERL circuits operated as quick as typical two-phase energy recovery logic counterparts. HSPICE simulation with an 8bit logarithmic Look ahead Adder (LLA) employing static CMOS,CAL (an existing single-phase primarily 


\section{International Journal of Recent Trends in Electrical \& Electronics Engg., Apr., 2018. OIJRTE \\ ISSN: 22316612}

based energy recovery logic), and QSSERL showed that the QSSERL adder consumes solely fifty six of energy like its static CMOS counterpart at $10 \mathrm{MHz}$ and achieves higher energy potency than CAL [82]. $\mathrm{Xu}$ Jian etal suggested a Research of Adiabatic Multiplier Based on CTGAL. They planned a brand new adiabatic multiplexer supported by the investigation of Clocked Transmission Gate adiabatic Logic (CTGAL) circuit, in their paper. It comprises of a halfway item generator, a fractional item compressor and a parallel prefix snake. CTGAL is employed altogether the circuits to charge and discharge the node capacitances while not the threshold value losing and therefore the charge on the output node capacitances are often recovered fully. Therefore the power consumption of the freshly designed circuits is considerably reduced [26].

M. Hempstead proposed an application specific design that integrates an event processor that assists main microcontroller executing needed system tasks. The given approach guarantees smart power improvement; however, no real world implementation results are given [27]. The approach in [28] utilizes hardware acceleration and optimized radio in a highly integrated single-chip solution. It applies an 8- bit data, 16-bit instruction CPU with reported size of $0.381 \mathrm{~mm} 2$ in a $0.25 \mathrm{um}$ process. The reduction of power in sensing element node radio is additionally investigated. One novel approach to low power radio is given in Pico Radio project [29]. Some researchers propose use of wake-up radio so as to scale back the radio power. Wakeup radio serves as a low-power switch to the node transceiver [30]. The implementation of advanced power-saving techniques such as dynamic voltage scaling [31] and power gating [32] promises to deliver additional reduction of node power consumption. The energy harvest is additionally thought-about as a possible resolution to increase the battery life [33]. Employing an asynchronous processor within the style of a detector node is planned in [34]. Another solution is an asynchronous architecture of a sensor node presented in [35]. The later solution includes additionally an energy harvesting circuit. However, no implementation results for those solutions have been presented. Fully asynchronous design is troublesome to implement and it needs all peripherals to own committed asynchronous interface.

The idea of instruction set bound ASIPs is accepted within the technical literature. During a crisp summary of ASIP style problems [36] is given. The reviewed ASIP style flows are targeted at performance constraints and don't take under consideration the energy consumption of the implementation. Moreover, the represented style flows often separate ASIP architectural style space exploration from ASIP instruction set synthesis. Within the current work, these style steps are combined, as a result of the instruction set is viewed as an interface to the design with mutual dependencies. As a consequence, design and instruction set are conjointly optimized so as to get optimum results. There are numerous ASIP style tools for the whole ASIP style flow from application to implementation. In the PEAS [37] style surroundings is represented that creates a direction set reenactment display and a synthesizable model from a design processor portrayal. The MetaCore DSP development system [38] is an ASIP style tool that supports style space exploration and design generation. Within the style flow, the development tools like compiler, assembler, and ISA machine likewise because the HDL description of the processor are generated. In [39] the ISDL machine description language is employed to come up with a little true instruction level machine and a synthesizable Verilog processor description. There are some style tools given within the literature that specialize in a set of the ASIP style flow. A framework for Compiler-ASIP co-design with feedback from an optimizing compiler to the ASIP style is represented in [40]. In [41] the RECORD compiler is given that uses a structural RTL model of a DSP as a start line of the compiler generation. In [42] authors proposed an extremely economical processor style methodology supported by the LISA 2.0 language. Generally the design style section is dominated by a repetitive processor model refinement based on the results of hardware and code simulation and identification.

\section{CONCLUSION \& FUTURE WORK}

Adiabatic Switching circuits are finding widespread use in day to day life. The efficiency, lifetime\& reliability of the circuit depend upon power consumption. If an adiabatic switching circuit is designed it will not only decrease the power requirement, but also conserve the circuit energy rather than dissipating it as heat. 
a. By designing SRAM based on Adiabatic Switching circuits designers will be able to achieve following:

- Power dissipation is the main constrain when it comes to Portability. The mobile device consumer demands more features and extended battery life at a lower cost.

- About $70 \%$ of users demand longer talk and stand-by time as primary mobile phone feature. Top $3 \mathrm{G}$ requirement for operators is power efficiency.

- Customers want smaller \& sleeker mobile devices. This requires high levels of Silicon integration in advanced processes, but advanced processes have low power efficiency which can be optimized by SRAM based on Adiabatic Switching circuits.

- The growing market of portable (e.g., cellular phones, gaming consoles, etc.), battery-powered electronic systems demands SRAM design with ultra-low power dissipation.

- As the integration, size, and complexity of the chips continue to increase, the difficulty in providing adequate cooling might either add significant cost or limit the functionality of the computing systems which make use of SRAM based on Adiabatic Switching circuits.

b. Effective SRAM based on Adiabatic Switching circuits increases optimization at all design abstraction layers as:

- System: Partitioning, Power down

- Algorithm: Complexity, Concurrency, Regularity

- Architecture: Parallelism, Pipelining, Redundancy, Data Encoding

- Circuit Logic: Logic Styles, Energy Recovery, Transistor Sizing

- Technology: Threshold Reduction, Multi-threshold Devices.

c. Helps firms monetize their innovations and grow by improving:

- Packaging and Cooling costs.

- Digital noise immunity.

- Battery life (in portable systems)

\section{d. Helps small and medium enterprises (SMEs)}

- Custom designed SRAM based on Adiabatic Switching circuits will help small and medium enterprises, design cost effective, highly reliable and environment friendly devices requiring energy efficient storage elements.

\section{e. Benefits consumers and society}

- SRAM based on Adiabatic Switching circuits provide consumers with innovative products and services in virtually every area of life, and helps protect consumers from low performance power hungry products.

- Improved battery life hence better performance, value for money, reliability etc.

\section{REFERENCES}

- Environmental concerns.

[1]. Deepti Shinghal, Amit Saxena, Dr. Arti Noor, Adiabatic Logic Circuits : A Retrospect, MIT International Journal of Electronics \& Communication Engineering(MIT IJ EC), ISSN : 2230-7664, Vol. 3, No. 2, August 2013, pp. 108-114. 


\section{International Journal of Recent Trends in Electrical \& Electronics Engg., Apr., 2018. CIJRTE ISSN: 22316612}

[2]. Amit Saxena, Deepti Shinghal, Arti Noor, Power Efficient Adiabatic Switching Circuits, MIT International Journal of Electronics \& Communication Engineering, Vol. 3, No. 2, pp. 98-103, August 2013.

[3]. Amit Saxena, Deepti Shinghal, Arti Noor, Comparative Analysis of Conventional CMOS \& Adiabatic Logic Gates, MITIJEC, Vol 4 No. 1, Jan 2014, pp.39-43.

[4]. Deepti Shinghal, Amit Saxena, Arti Noor, Kshitij Shinghal, Low Power Adiabatic Switching Circuits - A Review, International Conference on Advances in Electrical Electronics \& Computer Engineering (ICAEECE 2014) at MIT, Moradabad, March 8-9, 2014.

[5]. Amit Saxena, Deepti Shinghal, Dr. Arti Noor, Kshitij Shinghal, A Review of Energy Dissipation for Adiabatic Switching of CMOS based Logic Circuits, International Conference on Advances in Electrical Electronics \& Computer Engineering (ICAEECE 2014) at MIT, Moradabad, March 8-9, 2014.

[6]. Deepti Shinghal, Pragati Gupta, Aastha, Devendra Singh, Komal Sharma, Comparative Analysis of Adiabatic NAND Gate, International Conference on Advances in Electrical Electronics \& Computer Engineering (ICAEECE 2014) at MIT, Moradabad, March 8-9, 2014.

[7]. Amit Saxena, Deepti Shinghal, Kshitij Shinghal, Design and Implementation of Adiabatic based Low Power Logic Circuits, International Research Journal of Engineering and Technology (IRJET), Volume: 02 Issue: 02, pp. 498-504, May-2015.

[8]. Deepti Shinghal, A.N. Mishra, Amit Saxena, Design and Implementation of Adiabatic Latch for Low Power Embedded Systems, International Journal of Scientific Research and Management Studies (IJSRMS), Volume 2 Issue 4, July 2015, pg: 230-236. doi: 10.7323/ijsrms/v2_i4_8

[9]. Deepti Shinghal, A.N. Mishra, Farooq Hussain, Amit Saxena, Low Power Architecture for ASIP's: Based on Adiabatic Switching Principles, International Journal of Engineering Sciences \& Emerging Technologies, Volume 8, Issue 6, May 2016, pp: 290-297. doi: 10.7323/ijeset/v8_i6/05

[10]. Amit Saxena, Kshitij Shinghal, Deepti Shinghal, An Efficient Adiabatic Switching Circuit Design for Low Power Applications, International Journal of Engineering Sciences \& Emerging Technologies, Volume 8, Issue 6, May 2016, pp: 282-289. doi: 10.7323/ijeset/v8_i6/04

[11]. Amit Saxena, Kshitij Shinghal, Deepti Shinghal, "Adiabatic SRAM for Low Power Devices", International Journal of Recent Trends in Electrical and Electronics Engineering (IJRTE), Volume 4 Issue 2, pp. 56-63, March 2017. doi: 10.7323/ijrte/v4_i2/03

[12]. P. Leroux et al., "Design and Assessment of a Circuit and Layout Level Radiation Hardened CMOS VCSEL Driver," in IEEE Transactions on Nuclear Science, vol. 54, no. 4, pp. 1055-1060, Aug. 2007.

[13]. D. Mateo and A. Rubio, "Design and implementation of a $5 \times 5$ trits multiplier in a quasi-adiabatic ternary CMOS logic," in IEEE Journal of Solid-State Circuits, vol. 33, no. 7, pp. 1111-1116, Jul 1998.

[14]. Joohee Kim, C. H. Ziesler and M. C. Papaefthymiou, "Energy recovering static memory," Low Power Electronics and Design, 2002. ISLPED '02. Proceedings of the 2002 International Symposium on, 2002, pp. 92-97.

[15]. Michael P. Frank, "Reversible Computing and Truly Adiabatic Circuits: The Next Great Challenge for Digital Engineering," invited talk presented at the Fifth IEEE Dallas Circuits and Systems Workshop on Design, Applications, Integration and Software (DCAS-06), held Oct. 29-30, 2006.

[16]. Prasad D Khandekar, Shaila Subbaraman, and Abhijit V. Chitre, "Implementation and Analysis of QuasiAdiabatic Inverters", Proceedings of International Multiconference of Engineers and omputer Scientists 2010 vol 2,IMECS 2010, March 17-19 2010, Hong- Kong.

[17]. Ke Wu, Song Jia, Zhongjian Chen and Xuewen Gan, "Implementation of low-voltage true-single-phaseclocking (TSPC) logic using bulk dynamic threshold MOS technique," 2005 6th International Conference on ASIC, Shanghai, 2005, pp. 158-162.

[18]. P. D. Khandekar and S. Subbaraman, "Low Power 2:1 MUX for Barrel Shifter," 2008 First International Conference on Emerging Trends in Engineering and Technology, Nagpur, Maharashtra, 2008, pp. 404407.

[19]. T. Kuroda and M. Hamada, "Low-power CMOS digital design with dual embedded adaptive power supplies," in IEEE Journal of Solid-State Circuits, vol. 35, no. 4, pp. 652-655, April 2000.

[20]. H. Mahmoodi-Meimand and A. Afzali-Kusha, "Low-power, low-noise adder design with pass-transistor adiabatic logic," Microelectronics, 2000. ICM 2000. Proceedings of the 12th International Conference on, Tehran, 2000, pp. 61-64.

[21]. Joonho Lim, Dong-Gyu Kim and Soo-Ik Chae, "NMOS reversible energy recovery logic for ultra-lowenergy applications," in IEEE Journal of Solid-State Circuits, vol. 35, no. 6, pp. 865-875, June 2000.

[22]. B. Narasimham et al., "On-Chip Characterization of Single-Event Transient Pulsewidths," in IEEE Transactions on Device and Materials Reliability, vol. 6, no. 4, pp. 542-549, Dec. 2006. 


\section{International Journal of Recent Trends in Electrical \& Electronics Engg., Apr., 2018. CIJRTE \\ ISSN: 22316612}

[23]. Samik Samanta, "Power Efficient VLSI Inverter Design using Adiabatic Logic and Estimation of Power dissipation using VLSI-EDA Tool”, Special Issue of IJCCT Vol. 2 Issue 2, 3, 4; 2010 for International Conference [ICCT-2010], 3rd-5th December 2010.

[24]. Massimo Alioto and Gaetano Palumbo, Power Estimation in Adiabatic Circuits: A Simple and Accurate Model, IEEE Transactions on Very Large Scale Integration (VLSI) Systems, VOL. 9, NO. 5, October 2001.

[25]. S. Li, F. Zhou, C. Chen, H. Chen and Y. Wu, "Quasi-Static Energy Recovery Logic with Single PowerClock Supply," 2007 IEEE International Symposium on Circuits and Systems, New Orleans, LA, 2007, pp. 2124-2127.

[26]. Xu Jian, Wang Peng-jun and Zeng Xiao-yang, "Research of adiabatic multiplier based on CTGAL," 2007 7th International Conference on ASIC, Guilin, 2007, pp. 138-141.

[27]. M. Hempstead, N. Tripathi, P. Mauro, G.-Y. Wei, and D. Brooks, "An ultra-low power system architecture for sensor network applications," Proc. 32nd Annual International Symposium on Computer Architecture, Madison (USA) 2005, pp. 208-219.

[28]. J. M. Rabaey, J. Ammer, T. Karalar, S. Li, B. Otis, M. Sheets, and T.Tuan, "Pico Radios for wireless sensor networks: The next challenge in ultra-low-power design," Digest Tech. Papers IEEE International SolidState Circuits Conference, San Francisco (USA) 2002, pp. 200-201. 35

[29]. L. Gu and J. A. Stankovic, "Radio-triggered wake-up capability for sensor net- works," Proc. JOth IEEE Real-Time and Embedded Technology and Applications Symposium, Toronto (Canada) 2004, pp. 27-36. 36

[30]. T. Burd, T. Pering, A. Stratakos, and R. Brodersen, "A dynamic voltage scaled microprocessor system," IEEE J. Solid-State Circuits, vol. 35, pp. 1571- 1580,2000 37

[31]. G. Panic, D. Dietterle, Z. Stamenkovic, "Architecture of a Power-Gated Wireless Sensor Node," dsd, pp.844-849, 2008 lith EUROMICRO Conference on Digital System Design Architectures, Methods and Tools, 200838

[32]. R. Amirtharajah and A. P. Chandrakasan, "Self-powered signal processing using vibration-based power generation," IEEE J. Solid-State Circuits, vol. 33, pp. 687- 695, 1998. 39

[33]. C. Kelly, IV , V. Ekanayake , R. Manohar, SNAP: A Sensor-Network Asynchronous Processor, Proceedings of the 9th International Symposium on Asynchronous Circuits and Systems, p.24, May 1215, 2003. 40

[34]. Y. Ammar, A. Buhrig , M. Marzencki , 8. Charlot , S. Basrour , K. Matou, M. Renaudin, "Wireless sensor network node with asynchronous architecture and vibration harvesting micro power generator," Proceedings of the 2005 joint conference on Smart objects and ambient intelligence: innovative contextaware services: usages and technologies, October 12- 14,2005, Grenoble, France. 41

[35]. M. K. Jain, M. Balakrishnan and A. Kumar, "ASIP design methodologies: survey and issues," VLSI Design, 2001. Fourteenth International Conference on, Bangalore, 2001, pp. 76-81. 42

[36]. Jin-Hyuk Yang et al., "MetaCore: an application-specific programmable DSP development system," in IEEE Transactions on Very Large Scale Integration (VLSI) Systems, vol. 8, no. 2, pp. 173-183, April 2000. 44

[37]. P. Russo G. Hadjiyiannis and S. Devadas, "A methodology for accurate performance evaluation in architecture exploration”, New Orleans, 36th Design Automation Conference, June 1999.

[38]. A. Nicolau F. Onion and N. Dutt. Incorporating compiler feedback into the design of ASIPs, pages 508513. Proc. of European Design and Test Conference, 1995.

[39]. R. Leupers. Retargetable Code Generation for Digital Signal Processors. Kluwer Academic Publishers, 1997.

[40]. Hoffmann, A.; Fiedler, F.; Nohl, A.; Parupalli, S.; , "A methodology and tooling enabling application specific processor design," V LSI Design, 2005. 18th International Conference on, vol., no., pp. 399404, 3-7 Jan. 2005

[41]. Deepti Shrimal, Manoj Kumar Jain, "Instruction Customization: A Challenge in ASIP Realization", International Journal of Computer Applications (0975 - 8887) Volume 98- No.15, July 2014.

[42]. T. Glokler and H. Meyr, "Power reduction for ASIPS: a case study," Signal Processing Systems, 2001 IEEE Workshop on, Antwerp, 2001, pp. 235-246. 


\section{AUTHORS BIOGRAPHY}

Deepti Shinghal was born in Moradabad, Uttar Pradesh, India in January 1980. She received her Bachelor of Engineering (B.E.) degree in Electronics and Communication Engineering Rohilkhand University, Bareilly in 2000. Thereafter she worked for one year as a Faculty Member in the Department of Electronics and Communication Engineering at Aligarh Institute of Engineering \& Technology, Aligarh. Then she joined the Moradabad Institute of Technology, Moradabad in 2002. She received her M. Tech. degree in VLSI Design from U. P. Technical University, Lucknow in the year 2008.

Farooq Husain received the B.Sc. Engg. degree in Electronics and Communication engineering from Jamia Millia Islamia, New Delhi in 1993. \& M. Tech in Electronics Engineering (Specialization in Communication \& Information Systems) from A.M.U. Aligarh, 1999. He received Ph.D. in Electronics Engineering (Topic: Digital Watermarking of Multimedia Data) from A.M.U. Aligarh, 2013.

Shuchita Saxena received the Bachelor of Technology degree in Electronics and Communication engineering from Moradabad Institute of Technology, Moradabad in 2004. \& M. Tech in Microwave Engineering from Uttar Pradesh Technical University, Lucknow. Her main research interest is in analysis of microstrip antennas.

Amit Saxena has 12 Years of experience in the field of Academic. He obtained his Bachelor's degree in Electronics \& Communication Engineering from I.E.T., Rohilkhand University, Bareilly and Masters degree (VLSI Design) in 2009 from UPTU, Lucknow. He started his career from MIT, Moradabad. Presently he is working as an Assistant Professor, Deptt of E\&C Engg., at MIT Moradabad. He has published number of papers in international \& national journals, conferences and seminars. He is an active Member of Various Professional Societies such as ISTE, IACSIT, IAENG etc.

Kshitij Shinghal has 18 Years of experience in the field of Academic and is actively involved in research \& development activities. He obtained his Doctorate degree from Shobhit University Meerut in 2013, Masters degree (Digital Communication) in 2006 from UPTU, Lucknow. He started his career from MIT, Moradabad. Presently he is working as an Associate Professor, Deptt of E\&C Engg., at MIT Moradabad. He has published number of papers in national journals, conferences and seminars. He has guided two Masters, more than sixty students of B. Tech, and guiding three Ph.D. \& M. Tech. theses. He is an active Member of Various Professional Societies such as ISTE, IACSIT, IAENG etc.
